\title{
Factors Affecting Quality of Life among Elderly Population in Iran
}

\author{
Laleh Golamrej Eliasi ${ }^{1}$, Hamideh Addelyan Rasi ${ }^{2, ~}$, Arash Tavakoli ${ }^{2}$ \\ ${ }^{1}$ Department of Social Work, University of Social Welfare \& Rehabilitation Sciences, Tehran, Iran \\ ${ }^{2}$ Department of Social Work, Faculty of Social Sciences, Allameh Tabataba'i University, Tehran, Iran
}

Email address:

elyasy_laleh@yahoo.com (L. G. Eliasi), addelyan@atu.ac.ir(H. A. Rasi), arash.t67@gmail.com (A. Tavakoli)

${ }^{*}$ Corresponding author

\section{To cite this article:}

Laleh Golamrej Eliasi, Hamideh Addelyan Rasi, Arash Tavakoli. Factors Affecting Quality of Life among Elderly Population in Iran. Humanities and Social Sciences. Vol. 5, No. 1, 2017, pp. 26-30. doi: 10.11648/j.hss.20170501.15

Received: January 9, 2017; Accepted: February 6, 2017; Published: March 1, 2017

\begin{abstract}
This study aimed to find out the factors affecting older adults' quality of life in Tehran. The present study was included 380 respondents aged 60 and over. Standard quality of life questionnaire (WHOQOL - BREF) was used to collect the data. Correlation Coefficient Pearson, T-test, ANOVA and Spearman were used to assess the association between quality of life and its effective factors. Findings showed that high quality of life was associated with education, marital status, diseases, disabilities and housing however, there was not any association between age, gender and employment status with quality of life. The results of this study showed the effective factors on quality of life among elderly people in Tehran. Furthermore the findings propose to focus on social welfare policy through design proper instructional guidelines on quality of life in the elderly population, but further researches need to be conducted in this field.
\end{abstract}

Keywords: Aging, Quality of Life, Iran, Effective Factors

\section{Introduction}

Aging is a stage of life that is significantly different from the other ones [1]. Because of the decreasing birth rate and medical science advancement, the elderly population is increased day by day [2]. The United Nations in 2006 estimated the number of elderly people in the world was 700 million [3] and it is expected that this population will be doubled in the next 40 years (annual increasing of the elderly population in the world is $2.5 \%$ ). $52 \%$ of this population is in Asian countries and $40 \%$ is living in developing countries [4]. In Iran, according to general census in 2007, the population of people who were older than 60 was $7.27 \%$ of the total population. General census in Iran showed that the population of seniors in Tehran was 609283 [5]. The consequences of the aging, demand an increased focus on aging within the social work profession through working with older people and their families.

With respect to vulnerability in old age period in the life, aged people confront a number of difficulties which are related to their age and environment such as suffering from chronic diseases, loneliness and the lack of social protection and in many cases due to physical and mental disabilities, their independence is threatened. These problems lead to reduction the quality of life (QOL) (Molton \& Jensen, 2010; Gotay, Korn, McCabe, \& Moore, 1992).

QOL is a welfare state which includes 2 parts: the first one is an ability of doing daily activity that is physical, psychological and social health and the second one is satisfaction of performance, controlling and treatment of diseases (Gotay et al., 1992).

Many complex factors are involved in QOL so if seniors' conditions create an independent situation for them and they enable to play appropriate social roles, they will have a high QOL [6]. Due to this, innovative planning and policy reforms in developing and underdeveloped countries are required, to fulfill this goal, the World Health Organization consider a program which focuses on empowerment processes in three areas: elderly people and health improvement and welfare for elderly and assurance of empowering and supporting for elderly. The aged people are social capital for each country and QOL in the end of the human life cycle affects surpassing the life expectancy of every country population. Many studies 
have been done about this subject, but most of them were done in nursing home or just applied to some regions of Tehran. The present study covers all regions in Tehran and aimed to examine the association between QOL with gender, age, employment status, education, marital status, diseases, housing and disabilities among Iranian older people aged 60 years and over.

\section{Methods}

The present study is a survey study which was done on 380 aged citizens in Tehran ( $\geqq 60$ years). The sample size was determined by Cochrane formula. The senior population in Tehran is 609283 [5]. Using this formula, a sample size including 380 elderly individuals was chosen to participate in this study.

Sampling was done with considering Tehran position. Tehran is divided into 22 regions. In these regions, people have different socio-economic situations. We selected five regions of Tehran that showed all segments of society. These clusters were chosen from north, south, east, west and center of Tehran. In the specified clusters, samples were randomly selected (cluster random sampling). Participants, seniors who spent their leisure times in public parks, were selected from the parks in the five different regions of Tehran.

For data collection, a demographic questionnaire was applied to collect some variables such as gender, age, marital status, employment status, education, disabilities, diseases and housing situation. In addition we used The World Health Organization Quality of Life Assessment - Brief Instrument (WHOQOL - BREF) to evaluate QOL [7]. The questionnaire contains 4 scales. These scales included physical, psychological, social relationship and environmental domains. Physical domain included subjects about daily activities, amount of energy to perform daily activities and quality of sleep. Psychological domain included amount of concentration on activities, life and physical satisfaction, personal fulfillment and hopelessness. Social relationships domain was about sexual and personal relationships. Environmental part included subjects such as safety, access to environmental information, public transport and health services. All scales had the same points. Each scale was rated from 1 to 5: 1 indicating the worst and 5 indicating the best conditions [8]. The Iranian version of the WHOQOL-BREF has recently been validated, displaying that the social relationship dimension had uncertain reliability [7].

In present study the data was analyzed through using, SPSS statistics 18 to apply Correlation Coefficient Pearson, T-test, ANOVA and Spearman test in order to investigate the relationship between the variables. We considered $95 \%$ confidence interval in the study.

\section{Result}

In this study, total participants were 380; 201 men (52.89\%) and 179 women $(47.11 \%)$. In terms of employment status, 149 people were employed (39.21\%) and 231 people were unemployed (60.79\%). 48 people were disable (12.63\%), for walking they used wheelchair or walker, and 332 people were not disable (87.37\%). We used T-test to assess the relationship between QOL and these 3 variables; gender, employment status, and disabilities. (Table 1) There was only the association between QOL and disability situation (P-value = $0.05)$

Table 1. The relationship between $Q O L$ and gender, employment status and disabilities.

\begin{tabular}{llll}
\hline Variable & mean $( \pm$ SD $)$ & p-value & t \\
\hline Gender & & & \\
Female & $81.20( \pm 9.33)$ & 0.141 & -1.48 \\
Male & $78.33( \pm 10.21)$ & & \\
Employment & & & \\
Yes & $81.57( \pm 11.37)$ & & \\
No & $78.5( \pm 8.39)$ & 0.105 & 1.63 \\
Disability & & & \\
Yes & $73.16( \pm 9.31)$ & 0.023 & -2.53 \\
No & $80.45( \pm 9.57)$ & & \\
\hline
\end{tabular}

In terms of marital status 27 people were single (7.1\%), 239 people were married $(62.89 \%), 68$ were widowed (17.89) and 46 were divorced (12.12\%). In terms of diseases, 293 people answered they had specific disease $(77.10 \%)$ and 87 people answered they had not have any disease $(22.90 \%)$.

The details are as follows, 133 people had heart disease (35\%), 31 people had Diabetes $(8.16 \%), 32$ people had kidney disease $(8.42 \%), 25$ people had respiratory disease $(6.58 \%)$ and 72 people had Musculoskeletal diseases (18.94\%). In terms of housing, 189 people were owner-occupiers (49.73\%), 90 people lived in leased house (23.68\%), 49 people lived with their children $(12.89 \%), 7$ people lived with other relatives $(1.84 \%)$ and 45 people lived in different situation (such as they lived in their work place or other) $(11.84 \%)$. (Table 2) ANOVA test was used to evaluate the relationship between QOL and these 3 factors.) There were the association between QOL and marital status, diseases and housing ( $\mathrm{P}$-value $=$ $0.05)$.

Table 2. The relationship between quality of life and marital status, diseases and housing.

\begin{tabular}{lll}
\hline Variable & F & P-value \\
\hline Marital status & & \\
Single & & \\
Married & 2.87 & 0.005 \\
Widowed & & \\
Divorced & & \\
Diseases & & \\
Heart diseases & \\
Diabetes & & \\
Kidney diseases & & \\
Musculoskeletal & & \\
Respiratory diseases & 3.025 & \\
Housing & & \\
Private house & & \\
Leased house & & \\
Children's house & & \\
Relative's house & 3.657 & \\
\hline
\end{tabular}


The mean age of total participants was 69.8. 334 people were between 60 - 74 years old $(12.63 \%), 48$ people were between 75 - 90 years old $(87.37 \%)$ and nobody were older than 90. In order to measure the relationship between QOL with age, we used Correlation Coefficient Pearson. There was not any association between them. (Table 3)

Table 3. The relationship between $Q O L$ and age.

\begin{tabular}{lll}
\hline Variable & Pearson Correlation & P-value \\
\hline Age & & \\
$60-74$ & & \\
$75-90$ & 0.172 & 0.31 \\
$>90$ & & \\
\hline
\end{tabular}

In terms of education, 182 people were illiterate or they had primary education $(47.89 \%), 174$ people had diploma or bachelor (45.78\%) and 24 people had master or PhD (6.33\%). We used Spearman correlation to estimate the association between QOL and education. There was not any association between them. (Table 4)

Table 4. The relationship between $Q O L$ and education.

\begin{tabular}{lll}
\hline Variable & Spearman correlation & P-value \\
\hline $\begin{array}{l}\text { Education } \\
<\text { diploma }\end{array}$ & \\
$\begin{array}{l}\text { Diploma- bachelor } \\
\text { Master - PhD }\end{array}$ & 0.281 & 0.32 \\
\hline
\end{tabular}

\section{Discussion}

In this study, we aimed to assess the association of QOL with the effective factors such as gender, age, employment status, education, marital status, diseases, housing and disabilities among Iranian elderly people. The results showed that gender had no effect on quality of life. This finding also has been confirmed by some studies [9, 10]. But some previous studies on older people's QOL which have been done in Iran [11] or in other countries [12, 13] showed that men had higher QOL compared with women. Perhaps due to Iranian tradition and culture, for instance women should be at home and breed their children, so QOL among women is less than men, but Tehran has a different situation regarding the culture and values, most women in Tehran are engaged in some forms of employment and they work outside of the home and this situation would be affected on their QOL.

In terms of age, between different age groups, significant correlations have not been found and this is in line with Habibi's study that showed there is not any connection between age and QOL [14] however, various studies have noted that there is a direct correlation between aging and decreased QOL [15-17]. This difference maybe was related to participants' physical health in this study. All participants spent their days mostly at the park since their physical health let them to do such physical activities. They had similar situations regarding physical function and activities, consequently the result showed no connection between age and QOL among the participants.

The other variable was investigated in this study, was education. The results showed that QOL is not much different in the first two groups (Table 4), but QOL was higher among participants with a master or $\mathrm{PhD}$ degree than the other two groups. Previous studies on QOL among older adults had also shown a direct relationship between education and QOL [15, 18]. However, some researches indicated that there is no relationship between education and QOL and some studies showed there is an inverse relation $[19,20]$. People who have low education are less familiar with problem solving methods and positive coping strategies. These people have poor socio-economic situations that cause more stress and lower QOL among them [21].

In present study, there was no connection between employment and QOL. Current employment was considered in this study and we did not focus on the previous job. Other studies indicated that income is directly related to QOL [22, 23]. Marital status was another factor that was assessed in the study. Results showed that married people had higher QOL in comparison to the other group. This finding is consistent with those of previous studies $[15,24,25]$. One possible explanation for this result is that married people can improve QOL compared with those who are single, divorced, or bereaved due to the social relationship with the spouse that causes protective and supportive effects. However, some study reported that there is no difference between married, widowed, divorced and single aged people [14, 26].

In present study, the relationship between different diseases like diabetes, heart disease, respiratory, musculoskeletal, and kidney diseases and QOL was evaluated. According to the results, people who suffered from diabetes had more QOL compared with others and people with heart and kidney diseases had less QOL. Other studies indicated that disease affects on QOL, but they did not mention which kind of disease has more influence on QOL [27, 28]. With regard to disabilities, there was a direct connection between disabilities and QOL. The finding is consistent with some previous studies [27, 29]. The result of this study is also in line with that of Bindawas, et al, [30] indicated that increased levels of lower extremity performance was associated with slower rates of decline in physical and mental health-related QOL among older Mexican Americans. In present study, the housing situation had an impact on the QOL. Among house owners, QOL was more than lease holders and lease holders had a higher QOL compared with the other groups. The result is in line with Alipour, et al, [11].

\section{Conclusion}

In conclusion, this study suggests working on social welfare policy with the aim of improving and extending QOL among older Iranians. Some effective programs can be considered in the aged population like periodic checkups, extending the aging clinics, improving health insurance and welfare facilities. In addition, educational programs based on health promoting behaviors in the elderly can affect on the health and QOL among older population. There are many aging problems that are result from unhealthy lifestyles that can be addressed 
by the policy and decision makers of the health system through focusing on successful aging and adopting health promoting behaviors that can prevent and decrease aging problems and the financial burden and related costs. In fact, when the knowledge and competency on the physical, mental, and nutritional needs, among the elderly increase, they can adapt themselves to the aging better consequently, aging can be postponed or its problems can be decreased and finally health and QOL are increased.

As a result, we recommend that health promoters and practitioners to focus on designing and implementing effective interventions to educate people about ageing and related issues like ageing disease, disabilities and as well as preventive methods in order to decrease the ageing consequences. In addition, psychosocial interventions with focusing on expanding social relationship and social support can decrease social isolation in the elderly and consequently improve QOL.

Long term recommendations in welfare policy could be involved changing attitudes about ageing in the society, promoting intergenerational programs and creating supportive social services such as elder abuse and neglect prevention services.

For further research in this area, we suggest to study on social health and the effect of social networks and social support on elderly population's QOL in different parts of Iran.

\section{References}

[1] M. I. Lapid, K. M. Piderman, S. M. Ryan, K. J. Somers, M. M. Clark, and T. A. Rummans, "Improvement of quality of life in hospitalized depressed elderly," Int Psychogeriatr, vol. 23, pp. 485-98, 2011.

[2] L. M. G. Paskulin, M. Aires, A. V. Goncales, C. C. B. Kottwitz, E. P. Morais, and M. A. Brondani, "Aging diversity, and health: the Brazilian and the Canadian context," Act Paul Enferm, vol. 24, pp. 851-56, 2011.

[3] United Nations Population Division, "World Population Aging," New York, 2007.

[4] World Health Organization (WHO), "Global health expectancy research among older people," Aging and health and technical report series, 10, 2002.

[5] H. Sajadi, A. Biglarian, "Quality of life elderly women in a nursing home Kahrizak charity," Payesh Health Monit, vol. 6, pp. 105-8, 2007. (Persian).

[6] T. P. Ng, B. F. Broekman, M. Niti, X. Gwee, and E. Kua, "Determinants of successful aging using a multidimensional definition among Chinese elderly in Singapore," Am J Geriatr Psychiatry, vol. 17, pp. 407-416, 2009. Doi: 10.1097/JGP.0b013e31819a808e.

[7] S. Nejat, A. Montazeri, K. Holakouie Naieni, k. Mohammad, and S. R. Majdzadeh, (2007). "The World Health Organization quality of Life (WHOQOL-BREF) questionnaire Translation and validation study of the Iranian version," Sci J Sch Public Health Institute of Public Health Research, vol. 4 (4), pp. 1-12, 2007.

[8] World Health Organization (WHO), "WHOQOL-BREF,
Introduction, administration, scoring and generic version of the assessment," Geneva: WHO, 1996. http://www.who.int/mental health/media/en/76.pdf.

[9] P. Assantachai, and N. Maranetra, "Nationwide survey of the health status and quality of life of elderly Thais attending clubs for the elderly." Journal of Thai Medical Association, vol. 86, pp. 938-46, 2003.

[10] Y. Lee, and S. Shinkai, "A comparison of correlates of self-rated health and functional disability of older persons in the Far East: Japan and Korea," Archive of Gerontology of Geriatrics, vol. 37, pp. 63-76, 2003.

[11] F. Alipour, H. Sajadi, A. Fruzan, and A. Biglarian, "The role of social support on quality of life for elderly," Social Welfare Quarter. 33, 147-65, 2009. (Persian).

[12] S. Canbaz, S. A. Tevfik, and S. Dabak, "The prevalence of chronic diseases and quality of life in elderly people in Samsun," Turk J Med Sci, vol. 33, pp. 335-40, 2003.

[13] I. Sabbah, N. Drouby, S. Sabbah, N. Retel-Rude 1, and M. Mer cier, "Quality of Life in rural and urban populations in Lebanon using SF-36 health survey," Health Qual Life Outcomes, vol. 1, p. 30, 2003. DOI: 10.1186/1477-7525-1-30.

[14] A. Habibi, M. Nemadi-Vosoughi, S. Habibi, and M. Mohammadi, "Quality of Life and Prevalence of Chronic Illnesses among Elderly People: A Cross-Sectional Survey," Ardebil Journal of Health and Hygine, vol. 3, pp. 58-66, 2012. (Persian).

[15] T. W. Lee, I. S. Ko, and K. J. lee, "Health promotion behaviors and quality of life among community-dwelling elderly in Korea," International journal of nursing studies, vol. 49, pp. 129-137, 2005.

[16] M. Fortin, G. Bravo, C. Hudon, L. Lapointe, J. Almirall, and M. F. Dubois, "Relationship between multi morbidity and health-related quality of life of patients in primary care," Qual Life Res, vol. 15, pp. 83-91, 2006.

[17] E. L. Garcia, J. R. Banegas, A. G. Perez-Regadera, R. H. Cabrera, and f. Rodriguez-Artalejo, "Social network and health-related quality of life in older adults: a population-based study in Spain,” Qual Life Res, vol. 14, pp. 511-20, 2005.

[18] M. Ahangari, M. Kamali, and M. Arjmand Hesabi, "Quality of elderly's life in Tehran senile culture house clubs," Salmand, vol. 2, pp. 182-9, 2007. (Persian).

[19] F. Ahmadi, A. Salar, and S. Faghihzadeh, "Quality of life in Zahedan elderly population," Hayat, vol. 10, pp. 61-7, 2004. (Persian).

[20] N. E. Avis, S. F. Assmann, H. M. Kravitz, P. A. Ganz, and M. Ory, "Quality of life in diverse groups of midlife women: assessing the influence of menopause, health status and psychosocial and demographic factors," Quality of life research: an international journal of quality of life aspects of treatment, care and rehabilitation. Vol. 13, pp. 933-46, 2004.

[21] E. M. Friedman, G. D. Love, M. A. Rosenkranz, H. L. Urry, R. J. Davison, and B. H. Singer, "Socioeconomic status predicts objective and subjective sleep quality in aging women," Psychosom Med, vol. 69, pp. 682-91, 2007.

[22] M. R. Bazrafshan, M. A. Hosseini, M. Rahgozar, and S. B. Sadat Maddah, "The effect of exercise on quality of life of elderly women of Jahandidegan Club Foundation, Shiraz, 2007,” HBI Online, vol. 1, p. 43, 2011. 
[23] A. Agha nouri, M. Mahmoudi, H. Salehi, and K. Jafarian, "Quality of life in the elderly people covered by health centers in the urban areas of Markazi Province, Iran," Iranian Journal of Ageing, vol. 6, pp. 20-9, 2012. (Persian).

[24] M. R. Bazrafshan, M. A. Hosseini, M. Rahgozar, and S. B. Sadat Maddah, "Quality of elderly's life in shiraz, Jahandidegan club," Iranian Journal of Ageing, pp. 333-41, 2008.

[25] L. Hyttinen, P. Kekalainen, A. F. Vuorio, H. Sintonen and T. E. Strandberg, "Health-related quality of life in elderly patients with familial hypercholesterolemia," International journal of technology assessment in health care, vol. 24, pp. 228-34, 2008.

[26] M. G. Lima, M. B. Barros, C. L. Cesar, M. Goldbaum, L. Carandina, and R. M. Ciconelli, "Health related quality of life among the elderly: a population-based study using SF-36 survey," Cadernos desaude publica, vol. 25, pp. 2159-67, 2009.

[27] J. M. Lyness, "End-of-life care: issues relevant to the geriatric psychiatrist," American J Geriatr Psych, vol. 12, p. 457, 2004.

[28] M. Matsuo, J. Nagasawa, A. Yoshino, K. Hiramatsu, and K. kurashik, "Effects of activity Participation of the elderly on quality of life," yonago acta medica, vol. 46, pp. 17-24, 2003.

[29] Y. Hellstrom, and I. R. Hallberg, "Determinants and characteristic of help provision for elderly people living at home and in relation on quality of life," Scandinavian Journal of Caring Science, vol. 18, pp. 387-95, 2004.

[30] S. M. Bindawas, A. J. Ottenbacher, J. Graham, E. Protas, K. S. Markides, and K. J. Ottenbacher, "Association Between Lower Extremity Performance and Health-Related Quality of Life in Elderly Mexican Americans," J Aging Health, vol. 27, pp. 1026-1045, 2015 